\title{
Application of reflectance spectroscopy to the estimation of uric acid, urea and glucose: an evaluation of the Ames Seralyzer
}

\author{
JFSTEVENS*, RG NEWALL† \\ From the Department of Chemical Pathology, Middlesex Hospital Medical School, London and †Miles Laboratories, \\ Stoke Poges, Slough
}

SUMMARY An original approach to the measurement of analytes in clinical chemistry has now become available, in which dry reagent strip technology is linked to measurement by reflectance spectroscopy. The present studies have evaluated the performance of the first of these test systems - for uric acid, urea and glucose, in serum - by comparison with conventional liquid chemistry methods. Satisfactory performance in terms of both precision and accuracy was obtained for all three test systems, the current "state-of-the-art" performance criteria being met; the Seralyzer system proved reliable and easy to use in the hands of trained operators. It should find a place as a "Stat" analyser in the laboratory, in specified wards and in Health Centres.

Traditionally, spectroscopy in analytical clinical chemistry is regarded as a transmission technique, in which light is allowed to pass through a coloured solution: the difference in intensity between the incident and transmitted light is a function of the concentration of the substance in solution producing the colour. This approach naturally requires the use of liquid reagents and liquid handling devices, with the inherent restrictions imposed by stability problems.

There is currently an increasing tendency away from complex multiparametric analysers, and towards more discrete analysers, both in and beyond the central laboratory. In keeping with this trend, "dry" chemistry methods are being developed, using either multilayer film technology or immobilised strip reagents impregnated into a cellulose matrix. The latter have been in existence-and widespread use-for many years, in the form of reagent strips for semiquantitative urine and blood analysis. As a logical extension of this expertise, Ames Division has developed a strip technology in which complex mixtures of reagents-similar to those used in conventional "wet" chemistries-are incorporated into a cellulose matrix and plastic support. On contact with the specimen, sequential reactions take place on the pad, producing colour changes which can be measured in the ultraviolet or visible regions of the spectrum. These changes are monitored and measured by the technique of reflectance spectroscopy, using a unique system-the "Seralyzer."

*Present address: St Stephen's Hospital, Chelsea, London SW 10 Accepted for publication 11 August 1982
In the present studies, the Seralyzer system has been examined in order to evaluate the potential as a routine "Stat" analyser, in the laboratory.

The initial chemistries to be examined were uric acid (urate), urea and glucose. Other chemistries will be studied when available.

\section{Principles involved}

REFLECTANCE SPECTROSCOPY

Collimated light from a xenon source is allowed to fall onto the opaque surface of the reagent pad. Some of the light is absorbed by the chromogens in and below the surface layer. Surface absorption is also affected by the texture of the material, its effect as a light-scattering agent, and by the concentration of the light-absorbing material present.

Under the conditions prevailing on the strip, the reflectance approximates to the absolute diffuse reflectance of an infinitely thick layer of material $(R \infty)$. Under such conditions, reflectance is approximately related to concentrations by the Kubelka-Munk' equation:

$$
\{\mathrm{F}(\mathrm{R} \infty)\} \quad=\frac{(1-\mathrm{R} \infty)^{2}}{2 \mathrm{R} \infty} \frac{\mathrm{K}}{\mathrm{S}} \cdot=\frac{\epsilon \mathrm{C}}{\mathrm{S}}
$$

where $(R \infty)=$ absolute diffuse reflectance of an infinitely thick layer of material.

$\epsilon=$ extinction coefficient.

$\mathrm{C}=$ concentration.

$\mathrm{K}=$ absorption coefficient.

$\mathrm{S}=$ scattering coefficient 
This is analogous to the Beer-Lambert law, for transmission spectroscopy.

Deviations from linearity occur at high concentrations and low reflectance values; reflectance measurements should be made between two concentration levels within the limits of linearity, and a two-point calibration utilised.

A more detailed review is given by Greyson.?

INSTRUMENTATION

Simple reflectance meters rely on the measurement of reflected light at an angle to the incident light falling on the sample pad. This is relatively insensitive, since only part of the reflected light is used; in addition, some of the reflected light may, due to surface reflection, not be involved in the absorption process, thus causing further errors.

A development from this relatively basic measurement technique utilised the integrating sphere: reflection and absorption occur inside a sphere with a highly reflective surface, thereby increasing the amount of light reaching the detector.

In the Seralyzer, the integrating sphere is coated with barium sulphate, and is illuminated by a xenon flash tube. The diffuse reflected light in the sphere is absorbed on encountering the sample/reagent pad; the change in intensity of the reflected light is compared to a baseline white reflector, and is measured as a ratio to a reference detector. The reflectance value so computed, is subsequently converted into units of concentration, by reference to the calibration curve.

THE SERALYZER SYSTEM

The Seralyzer system basically comprises a microprocessor reflectance photometer in association with dedicated solid phase reagent strips, as briefly outlined previously. The principles of the system are described by Zipp. ${ }^{3}$

The principal application of the Seralyzer is anticipated to be as a "Stat" analyser in the laboratory and in the Physician's office in areas of private medicine. Initially, the system has been qualified for the quantitative estimation of certain analytes in serum only; applicability for use with plasma specimens for each analytical parameter is being examined - brief initial observations are made in the present study.

A feature of the impregnated reagent strips employed is the requirement for complete saturation of the fibres by the specimen; hence, the specimen volume applied to the strip assumes importance. However, by virtue of this saturation, the strip response is essentially independent of specimen viscosity. Since very little residual water is present in the unused reagent strips, a considerable degree of stability is conferred, obviating the need for refrigeration.

Programmed modules or cartridges are supplied for each test parameter. These contain the correct filters for the chemical reactions taking place on the strip: instructions for calibration and test readings. and conversion to the appropriate concentration units: defined tolerance limits for acceptance of the calibration procedure: display of error codes. in the event of system faults: and a recognition system for the identification of the correct reagent strip for each test by means of bar codes on each strip. All tests are performed at a temperature of $37^{\circ} \mathrm{C}$. Tests are performed either as rate or end-point reactions. in the ultraviolet or visible regions of the spectrum.

OPERATION

Calibration is achieved by means of a two-point procedure. employing a Low and a High Calibration solution. This calibration will be accepted only within defined tolerance limits of slope. lincarity and reflectance.

For the three chemistries under investigation. 30 $\mu \mathrm{l}$ of serum which has been previously diluted threefold with distilled water. is applied to the reagent pad on the instrument table: air-displacement pipettes are supplied. The "Start" button is pressed, and the table is pushed fully in. After a preset time, the concentration appears on the digital display. Should sample concentrations lic beyond the linearity range of the system. the appropriate error code is displayed. Over-range samples may be diluted a further 1 in 3 with distilled water and retested. after activating the "dilute" key on the keyboard. The corrected concentration is then displayed.

In the present studies. the first three chemistries available have been examined-blood urea nitrogen (BUN). uric acid (urate) and glucose.

\section{Material and methods}

The Seralyzer instruments used were premarketing models; similarly, trial batches only of the reagent systems were used.

\section{CHEMICAL PRINCIPLES}

Urea (Blood urea nitrogen)

The test is based on the reaction of amides with $o$-phthalaldehyde. Urea reacts with $o$-phthalaldehyde to form 1,3-dihydroxyisoindoline (DHI). Under highly acidic conditions, the carbonium ion of the DHI reacts with an indicator, 3-hydroxy-1,2,3,4-tetrahydrobenzo(h)-quinoline (HTBQ), to form a blue chromogen. The acidic environment necessary for the test is provided by a cation exchange resin which has been incorporated into the paper. The overall chemical reaction is given below:

Urea $+o$-phthalaldehyde $\rightarrow 1,3$ dihydroxyisoindoline (DHI)

$\mathrm{DHI}+\mathrm{HTBQ} \frac{\text { cation exchange resin }}{(\mathrm{H}+\mathrm{form})}$ blue chromogen

This is a kinetic (rate) assay, performed at a wavelength of $620 \mathrm{~nm}$, with an incubation period of $70 \mathrm{~s}$. 
Uric acid

The Seralyzer uric acid test is based on the reaction in which hydrogen peroxide, generated by the uricase reaction, is measured by oxidative coupling of 3-methyl-2-benzothiazolinonehydrazone (MBTH) and primaquine diphosphate in the presence of peroxidase. The two separate reactions are outlined below:

$$
\begin{aligned}
& \text { Uric acid }+\mathrm{O}_{2}+\mathrm{H}_{2} \mathrm{O} \stackrel{\text { uricase }}{\longrightarrow} \text { Allantoin }+\mathrm{CO}_{2}+\mathrm{H}_{2} \mathrm{O}_{2} \\
& \mathrm{H}_{2} \mathrm{O}_{2}+\mathrm{MBTH}+\underset{\text { diphosphate }}{\stackrel{\text { Primaquine peroxidase }}{\longrightarrow}} \mathrm{H}_{2} \mathrm{O}+\text { coloured complex }
\end{aligned}
$$

This is an end-point reaction, measured at $560 \mathrm{~nm}$, with an incubation time of $135 \mathrm{~s}$.

\section{Glucose}

The glucose test utilises glucose oxidase to produce hydrogen peroxide and gluconolactone. The hydrogen peroxide so produced then oxidises $3,3^{\prime}, 5,5^{\prime}$ tetramethylbenzidine (TMB-reduced) in the presence of peroxidase to produce TMB (oxidised):

$$
\begin{array}{cc}
\text { Glucose }+\mathrm{O}_{2} \stackrel{\text { glucose oxidase }}{\longrightarrow} & \mathrm{H}_{2} \mathrm{O}_{2}+\text { Gluconolactone } \\
\mathrm{H}_{2} \mathrm{O}_{2}+\underset{\text { (reduced) }}{\mathrm{TMB}} \stackrel{\text { peroxidase }}{\longrightarrow} \mathrm{H}_{2} \mathrm{O}+\underset{\text { (oxidised) }}{\text { TMB }}
\end{array}
$$

The kinetic (rate) reaction is performed at $640 \mathrm{~nm}$, with an incubation time of 50-120 s.

\section{PROTOCOL}

Within- and between-run precision data were obtained using serum pools and commercial quality control sera at different analyte concentrations. Accuracy was assessed by the analysis of true clinical specimens on the Seralyzer. relative to the routine comparative laboratory methods. Replicate precision of the clinical specimen assays was also determined. The comparative laboratory methods employed were as follows:

(i) Urea $(B U N)$-Vickers $\mathrm{M} 300$ urease.

(ii) Uric acid-Vickers M300 uricase and AutoAnalyser-I phosphotungstic acid.

(iii) Glucose-Hitachi 706 glucose oxidase, and Beckman glucose oxidase electrode analyser.

\section{Results}

(i) Urea (BUN)

Precision data are shown in Tables 1 and 2; accuracy is depicted in Table 3 and Fig. 1. Precision of replicate clinical specimen assays is shown in Fig. 2.

(ii) Uric acid

Precision data are presented in Tables 4 and 5: accuracy data are shown in Table 6.

Table 1 Urea: within-run precision (expressed as $m g / d(B U N$ )

\section{(iii) Glucose}

Precision data are presented in Tables 7 and 8; correlation statistics are shown in Table 9.

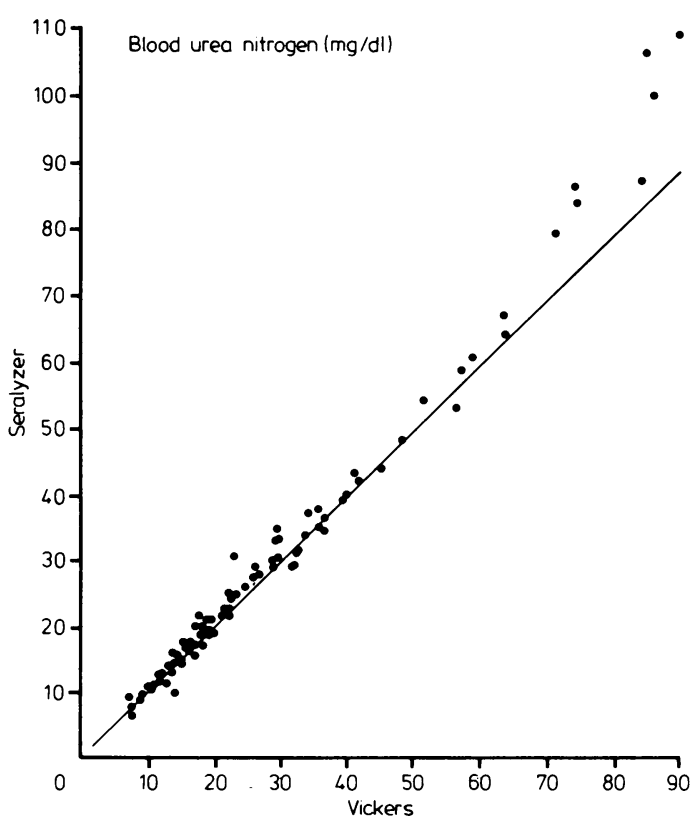

Fig I Correlation for BUN (Urea) between Seralvzer and

\begin{tabular}{|c|c|c|c|c|c|c|c|c|c|}
\hline \multirow[t]{2}{*}{$D a y$} & \multicolumn{3}{|c|}{ Lowlevel } & \multicolumn{3}{|c|}{ Medium level } & \multicolumn{3}{|c|}{ Highlevel } \\
\hline & Mean & $S D$ & $C V \%$ & Mean & $S D$ & $\mathrm{CV} \%$ & Mean & $S D$ & $C V \%$ \\
\hline 1 & $18 \cdot 2$ & $1 \cdot 08$ & $5 \cdot 9$ & $40 \cdot 1$ & $2 \cdot 51$ & $6 \cdot 3$ & $52 \cdot 8$ & 3.83 & $7 \cdot 2$ \\
\hline 2 & $18 \cdot 2$ & 0.98 & $5 \cdot 4$ & $41 \cdot 0$ & $2 \cdot 70$ & $6 \cdot 6$ & $53 \cdot 3$ & $5 \cdot 30$ & 9.9 \\
\hline 3 & $18 \cdot 3$ & 0.96 & $5 \cdot 3$ & $41 \cdot 1$ & $1 \cdot 76$ & $4 \cdot 3$ & $52 \cdot 8$ & 3.04 & $5 \cdot 8$ \\
\hline
\end{tabular}
Vickers analisers

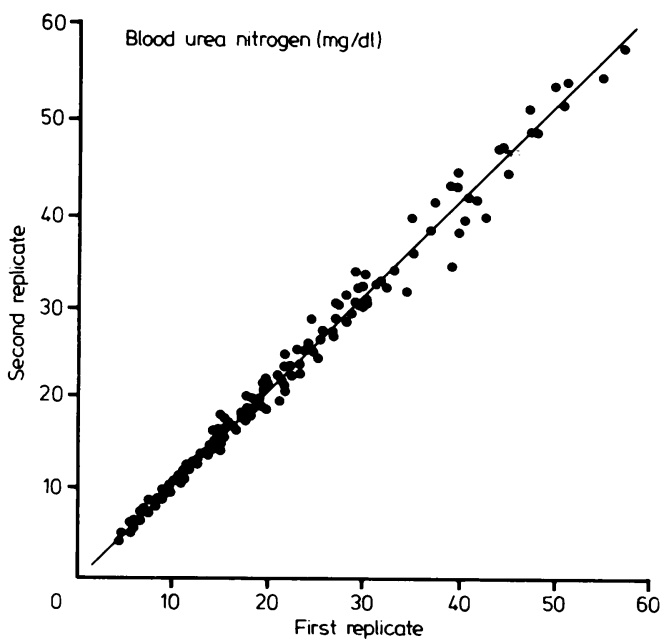

Fig 2 Precision of replicate clinical specimens on Seralyzer 
Table 2 Urea: between-run precision(Omega Control Sera I and II were assayed with each assay run of clinical specimens-13 runs)

\begin{tabular}{|c|c|c|c|c|}
\hline & \multicolumn{2}{|l|}{ Omegal } & \multicolumn{2}{|l|}{ Omega II } \\
\hline & Seralyzer & Vickers & Seralyzer & Vickers \\
\hline $\begin{array}{l}\text { Mean }(\mathrm{mg} / \mathrm{dl}) \\
\text { SD }(\mathrm{mg} / \mathrm{dl}) \\
\text { CV }(\%)\end{array}$ & $\begin{array}{c}14 \cdot 0 \\
0 \cdot 82 \\
5 \cdot 8\end{array}$ & $\begin{array}{l}13 \cdot 9 \\
0.67 \\
4 \cdot 8\end{array}$ & $\begin{array}{c}44 \cdot 1 \\
2 \cdot 15 \\
4 \cdot 9\end{array}$ & $\begin{array}{c}44 \cdot 7 \\
1 \cdot 71 \\
3 \cdot 8\end{array}$ \\
\hline
\end{tabular}

Table 3 Urea (BUN): comparison with routine laboratory method (accuracy)

\begin{tabular}{|c|c|c|c|c|c|}
\hline System & Specimen & $r$ & Slope & Intercept & $n$ \\
\hline Seralyzer/Vickers & Serum & 0.993 & $1 \cdot 195$ & -0.644 & 100 \\
\hline Seralyzer/Vickers & Plasma & 0.992 & 1.096 & -0.26 & 37 \\
\hline
\end{tabular}

Table 4 Uric acid: within-run precision data for Seralyzer (expressed as uric acid $m g / d l$ )

\begin{tabular}{|c|c|c|c|c|c|c|c|c|c|}
\hline \multirow[t]{2}{*}{$D a y$} & \multicolumn{3}{|c|}{ Low'level } & \multicolumn{3}{|c|}{ Medium level } & \multicolumn{3}{|c|}{ High level } \\
\hline & Mean & $S D$ & $C V \%$ & Mean & $S D$ & $C V \%$ & Mean & $S D$ & $C V \%$ \\
\hline 1 & $1 \cdot 66$ & $0 \cdot 12$ & $7 \cdot 3$ & $3 \cdot 84$ & 0.41 & $10 \cdot 8$ & $5 \cdot 45$ & 0.62 & $11 \cdot 3$ \\
\hline 2 & $1 \cdot 64$ & 0.09 & $5 \cdot 9$ & $3 \cdot 80$ & 0.33 & $8 \cdot 7$ & $5 \cdot 18$ & 0.33 & $6 \cdot 4$ \\
\hline 3 & 1.73 & 0.08 & $5 \cdot 0$ & 3.65 & 0.19 & $5 \cdot 2$ & $5 \cdot 38$ & $0 \cdot 26$ & $4 \cdot 8$ \\
\hline
\end{tabular}

Table 5 Uric acid: between-run precision

\begin{tabular}{|c|c|c|c|c|c|c|}
\hline & \multicolumn{2}{|c|}{ Seralyzer } & \multicolumn{2}{|c|}{ Vickers } & \multicolumn{2}{|l|}{$A A I$} \\
\hline & $I$ & II & 1 & II & $I$ & II \\
\hline $\begin{array}{l}\text { Mean (mg/dl) } \\
\text { SD (mg/dl) } \\
\text { CV }(\%)\end{array}$ & $\begin{array}{l}5 \cdot 2 \\
0 \cdot 31 \\
5 \cdot 9\end{array}$ & $\begin{array}{l}9 \cdot 7 \\
0 \cdot 44 \\
4 \cdot 5\end{array}$ & $\begin{array}{l}4 \cdot 2 \\
0 \cdot 10 \\
2 \cdot 3\end{array}$ & $\begin{array}{l}7 \cdot 9 \\
0 \cdot 17 \\
2 \cdot 2\end{array}$ & $\begin{array}{l}4 \cdot 6 \\
0 \cdot 12 \\
2 \cdot 5\end{array}$ & $\begin{array}{l}9 \cdot 2 \\
0 \cdot 38 \\
4 \cdot 1\end{array}$ \\
\hline
\end{tabular}

Table 6 Uric acid: comparison with routine laboratory method (accuracy)

\begin{tabular}{lllll}
\hline Systems & $r$ & Slope & Intercept & $n$ \\
\hline Seralyzer/Vickers & 0.972 & 0.989 & 0.04 & 105 \\
Seralyzer/AAI & 0.965 & 1.105 & -0.42 & 105 \\
\hline
\end{tabular}

Table 7 Glucose: within-run precision datafor Seralyzer (expressed in $\mathrm{mg} / \mathrm{dl}$ )

\begin{tabular}{|c|c|c|c|c|c|c|c|c|c|}
\hline \multirow[t]{2}{*}{$D a y$} & \multicolumn{3}{|c|}{ Lowpool } & \multicolumn{3}{|c|}{ Mediumpool } & \multicolumn{3}{|c|}{ Highpool } \\
\hline & Mean & $S D$ & $C V \%$ & Mean & $S D$ & $C V \%$ & Mean & $S D$ & $C V \%$ \\
\hline $\begin{array}{l}1 \\
2 \\
3\end{array}$ & $\begin{array}{l}83 \cdot 6 \\
85 \cdot 8 \\
86.6\end{array}$ & $\begin{array}{l}4 \cdot 1 \\
2 \cdot 5 \\
2 \cdot 6\end{array}$ & $\begin{array}{l}6 \cdot 5 \\
3 \cdot 8 \\
4 \cdot 6\end{array}$ & $\begin{array}{l}145 \cdot 1 \\
145 \cdot 3 \\
143 \cdot 3\end{array}$ & $\begin{array}{l}6 \cdot 5 \\
2 \cdot 4 \\
5 \cdot 1\end{array}$ & $\begin{array}{l}4 \cdot 5 \\
1 \cdot 6 \\
3 \cdot 5\end{array}$ & $\begin{array}{l}223 \cdot 6 \\
220 \cdot 1 \\
232 \cdot 9\end{array}$ & $\begin{array}{l}6 \cdot 5 \\
3 \cdot 8 \\
4 \cdot 6\end{array}$ & $\begin{array}{l}3 \cdot 1 \\
1.7 \\
1.9\end{array}$ \\
\hline
\end{tabular}

Table 8 Glucose: between-run precision (Control sera A and B assaved in triplicate with each run of clinical specimens)

\begin{tabular}{|c|c|c|c|c|}
\hline & \multicolumn{2}{|c|}{$A$} & \multicolumn{2}{|c|}{$B$} \\
\hline & Seralyzer & Hitachi & Seralyzer & Hitachi \\
\hline $\begin{array}{l}\text { No of runs } \\
\text { Mean (mg/dl) } \\
\text { SD }(\mathrm{mg} / \mathrm{dl}) \\
\text { CV }(\%)\end{array}$ & $\begin{array}{r}12 \\
96 \cdot 7 \\
4 \cdot 3 \\
4 \cdot 5\end{array}$ & $\begin{array}{l}12 \\
96 \cdot 4 \\
5 \cdot 1 \\
5 \cdot 3\end{array}$ & $\begin{array}{r}12 \\
261 \cdot 5 \\
11 \cdot 4 \\
4 \cdot 4\end{array}$ & $\begin{array}{c}12 \\
286 \cdot 8 \\
19 \cdot 1 \\
6 \cdot 7\end{array}$ \\
\hline
\end{tabular}


Table 9 Glucose: comparison with routine laboratory method (accuracy)

\begin{tabular}{llllr}
\hline System & $r$ & Slope & Intercept & $n$ \\
\hline Seralyzer/Hitachi & 0.975 & $0 \cdot 970$ & $2 \cdot 3$ & 168 \\
Seralyzer/Beckman & 0.989 & 0.985 & $10 \cdot 0$ & 31 \\
\hline
\end{tabular}

\section{Discussion}

Three chemistries have been examined and compared to existing, recognised laboratory methods, in terms of precision, accuracy and practical performance.

All results were expressed in units of $\mathrm{mg} / \mathrm{dl}$, and urea in terms of BUN, due to the unavailability of SI unit modules at this premarketing stage. Such modules are currently available in Europe. The Seralyzer urea system demonstrated an overall precision of $5.6 \% \mathrm{CV}$, which is perfectly acceptable and well within the critical $\mathrm{CV}$ of $7.4 \%$ as defined by Ross et $\mathrm{al} .{ }^{4}$ The results obtained from the different Seralyzer instruments gave good agreement with each other, and with the reference methodologies. In the UK, plasma may be preferred to serum by many laboratories for routine assays: the present studies demonstrate the acceptability of plasma for use with the Seralyzer urea system, although a slight but insignificant bias may exist. Correlation studies produced excellent linear regression parameters at BUN concentrations below $60 \mathrm{mg} / \mathrm{dl}$; above this concentration, further specimen dilution is required-this resulted in slightly poorer correlation. In the light of further experience, the urea (BUN) module has been modified by the manufacturers to correct for this slight dilution error at these very high concentrations.

The uric acid studies have all been performed on serum specimens; plasma cannot be used with the Seralyzer uric acid system, due to the reaction of heparin with the chromogen. The results show an overall precision of $6.0 \%$ $\mathrm{CV}$, which is again inside the criteria of Ross $\mathrm{et} \mathrm{al}^{4}(8.33 \%$ $\mathrm{CV})$. Comparison with the routine laboratory methods demonstrated a constant relationship. Nevertheless, the uric acid results obtained with the Seralyzer were very satisfactory for clinical useage.

The glucose results may. be expected to be examined more critically, in view of the long tradition of the Ames Division in blood glucose measurement. The overall coefficient of variation of $4.0 \%$ once more lies within the desired "state-of-the-art" criteria. Comparison with reference systems demonstrated good correlation in each case. A slight positive bias of the reference methodologies compared to the Seralyzer was demonstrated: this should be taken into account in practice-especially when assaying quality control materials. The Seralyzer will accurately measure glucose concentrations at levels of $35 \mathrm{mg} / \mathrm{dl}(1.9 \mathrm{mmol} / \mathrm{l})$ and above; cases of adult hypoglycaemia would be detected.
A comparison of these results with those obtained from a similar study in New York (A Karmen, personal communication 1982) using different comparative methodologies, showed equivalence in terms of accuracy and precision. This demonstrates the transportability of the Seralyzer system. In general, no significant examples of interference have been noted during the entire study. Excellent replicate precision of clinical specimens has been demonstrated for all three chemistries.

In conclusion, very encouraging results in terms of accuracy and precision have been obtained from this study, using reflectance spectroscopy in conjunction with dry chemistry systems. The requirement for sample dilution perhaps lessens the impact of the "dry" chemistry system; however, by the same token, potential interference is minimised by this dilution. Serum is currently the sole type of specimen qualified for use with the Seralyzer. We have shown that very satisfactory measurements of urea may be obtained using plasma samples; the suitability of plasma will be examined for future Seralyzer systems. The degree of expertise demanded by the air-displacement pipetting stages precludes the use of the Seralyzer by untrained or unskilled personnel, if optimal performance is to be obtained. It is therefore unsuitable for nursing use at the bedside. However, with appropriate technical expertise and complemented by a suitable quality control programme, the Seralyzer system could well find a place in Health Centres, GP centres, specified wards and notably, in the laboratory as a "Stat" system.

Grateful acknowledgements are due to the Courtauld Institute of Biochemistry, of the Middlesex Hospital Medical School, London; and Ames Division, Miles Laboratories Limited. Thanks are given to Marwin Elachker for his excellent technical assistance.

\section{References}

' Kubelka P. Munk FZ. Tech Physik 1931;12:593-601.

${ }^{2}$ Greyson J. Problems and possibilities of chemistry on dry reagent carriers. J Aut Chem 1981:3:66-70.

${ }^{3}$ Zipp A. Development of dry reagent chemistry for the clinical laboratory. $J$ Aut Chem 1981;3:71-4.

${ }^{4}$ Ross JW, Fraser MD, Moore TD. Analytic clinical laboratory precision-state of the art for thirty one analytes. Am J Clin Pathol 1980;74 suppl, 521-30.

Requests for reprints to: Dr JF Stevens, St Stephen's Hospital. Chelsea, London SW 10, England. 